\title{
Evaluation of laser range-finder mapping for agricultural spraying vehicles
}

\author{
Francisco-Angel Moreno ${ }^{1}$, Grzegorz Cielniak ${ }^{2}$ and Tom Duckett ${ }^{2}$ \\ 1 Department of System Engineering and Automation, University of Málaga, Spain \\ famoreno@uma.es \\ 2 Lincoln School of Computer Science, University of Lincoln, UK \\ \{gcielniak, tduckett\}@lincoln.ac.uk
}

\begin{abstract}
In this paper, we present a new application of laser rangefinder sensing to agricultural spraying vehicles. The current generation of spraying vehicles use automatic controllers to maintain the height of the sprayer booms above the crop. However, these control systems are typically based on ultrasonic sensors mounted on the booms, which limits the accuracy of the measurements and the response of the controller to changes in the terrain, resulting in a sub-optimal spraying process. To overcome these limitations, we propose to use a laser scanner, attached to the front of the sprayer's cabin, to scan the ground surface in front of the vehicle and to build a scrolling $3 \mathrm{~d}$ map of the terrain. We evaluate the proposed solution in a series of field tests, demonstrating that the approach provides a more detailed and accurate representation of the environment than the current sonar-based solution, and which can lead to the development of more efficient boom control systems.
\end{abstract}

Keywords: agri-robotics, 3d terrain reconstruction, outdoor mapping.

\section{Introduction}

Precision agriculture aims to utilise automated management and technology solutions for optimisation of various farming processes. Future agricultural systems will rely on machines performing tasks like ploughing, spraying or harvesting autonomously with minimal intervention from a human user. This work is concerned with a particular class of agricultural spraying vehicles, namely horizontal boom sprayers (see Figure 1). The modern generation of these vehicles feature adjustable spraying booms which can be automatically controlled to maintain a constant distance from the crops. This is a critical process as the height of the boom affects the amount and distribution of the sprayed substance, which has not only financial implications, but is also becoming increasingly important in the light of tougher environmental policies on the use of fertilisers and pesticides. The current boom control systems rely on ultrasonic sensors for measuring the height and level of the booms. The ultrasonic sensors, whilst inexpensive, are relatively slow and provide noisy information for only a small patch of the terrain immediately below the spraying boom. This results in limited effectiveness 


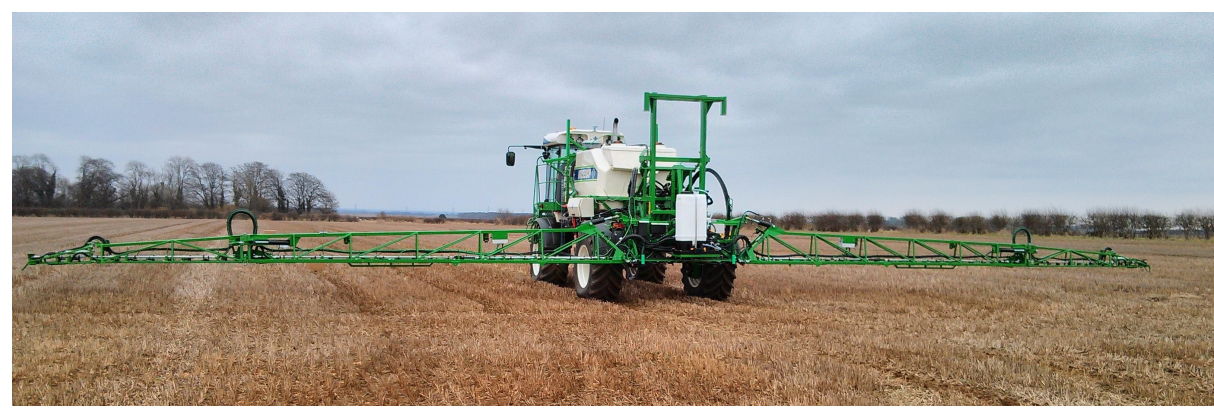

Fig. 1. A 24-meter wide sprayer model, Merlin 4000/24, by Househam Sprayers Ltd.

and restricts the maximum speed of the sprayer, since only a reactive control strategy is possible, resulting in a sub-optimal spraying process.

This paper investigates an alternative sensing technology based on laser range-finders (LRF) and terrain modelling that can provide predictive (i.e. with a longer "look-ahead"), higher-quality distance information. Laser range-finders, which have been widely used for over a decade in the mobile robotics community, have been applied to numerous applications such as mapping and navigation. Our proposal is to apply these well-established techniques to enhance the sensing abilities of agricultural spraying vehicles. The core component of the proposed system is a $3 \mathrm{~d}$ map of the terrain, reconstructed from a scanning laser range-finder and additional information provided by GPS. With this approach the terrain is sensed in advance, so the controller should have more time to adjust the height of the booms. The approach not only improves the accuracy of the provided distance information but can also enable new applications such as terrain-based vehicle steering or variable-rate spraying, leading towards development of fully autonomous spraying vehicles.

\section{Related Work}

Robotic applications in agriculture can bring numerous economic, societal and environmental benefits (e.g. reduced production costs, more friendly working environments, reduced contamination risks, etc.) [10]. However, the future development of such systems will have to address several challenges arising from the complexity of farming processes, outdoor environments, and the mechanical complexity and physical size of agricultural machinery. To meet these challenges, the future autonomous farming vehicles will have to rely not only on GPS-based solutions but also on a detailed representation of the environment, e.g. 3d maps.

The majority of outdoor 3d mapping applications consider urban environments (e.g. [9]) where there are physical, man-made structures that assist in the registration of $3 \mathrm{~d}$ scans, improving the quality of the resulting maps. Other examples include mapping solutions for off-road autonomous car driving [13], mining operations [5] and autonomous road inspection [8]. The latter system 
(RoadBot) fuses information from range scanners thanks to precise pose estimation obtained from high precision GPS (i.e. real-time-kinematics-enabled devices) with laser-aided height correction, resulting in a dense and precise map of the road. We assume a similar approach in our work. The majority of the presented mapping systems rely on laser scanners, although other sensors can be used, e.g. stereo vision [11].

Recent interest in agricultural robotics has resulted in several systems relying on $3 \mathrm{~d}$ respresentation of the envrionment. For example, [7] presents a multi-vehicle system for automating orchard farming operations like spraying and mowing. The vehicle navigation is performed by a combination of GPS, range and vision data, enabling obstacle avoidance and row-following behaviours. BoniRob [3] is a mobile phenotyping robotic platform equipped with a range of sensors designed to automatically measure different plant properties. The system functionality includes detection of the ground and individual plants based on 3d range maps and semantic place classification. The experiments conducted with different range sensing technologies suggest that the laser-based techniques are most suitable in the farming context.

Existing autonomous spraying solutions were mostly deployed in horticulture scenarios such as orchards or vineyards. For example,[16] describes techniques for building off-line 3d models of trees and their subsequent use for precision spraying. The models are based on probabilistic techniques that separate range data from ladar sensor into ground and tree canopy. The tree height and density is later used by a variable-rate spraying controller, resulting in more precise and directed application of the chemicals. Other examples include a review of different tree area estimation methods [14], 3d fluid dynamics modelling for improved spraying [6], and range-based real-time control of the spraying [15].

Related work in technological solutions for horizontal boom sprayers considers mostly mechanical aspects of the booms, including their design [2], analysis of oscillations [4], and control based on mechanical modelling with a simple infra-red distance sensing solution [12]. In contrast, our work concentrates on the novel application of laser range-finder sensing, together with GPS information, to build a scrolling $3 \mathrm{~d}$ model of the terrain, which could later be used for improving the control of the sprayer booms.

\section{System Description}

\subsection{Horizontal Boom Sprayer}

The main components of the current system consist of a spraying vehicle and an adjustable spraying boom which can be folded and unfolded for easier transportation and storage (see Fig. 1). The vehicle also carries a tank containing chemicals being distributed on the field from the nozzles located on the booms. The length of the booms depends on the sprayer model and ranges from 12 to 18 meters on each side. The boom is automatically controlled by a custom made controller which can adjust the height of the boom platform and the boom incline 


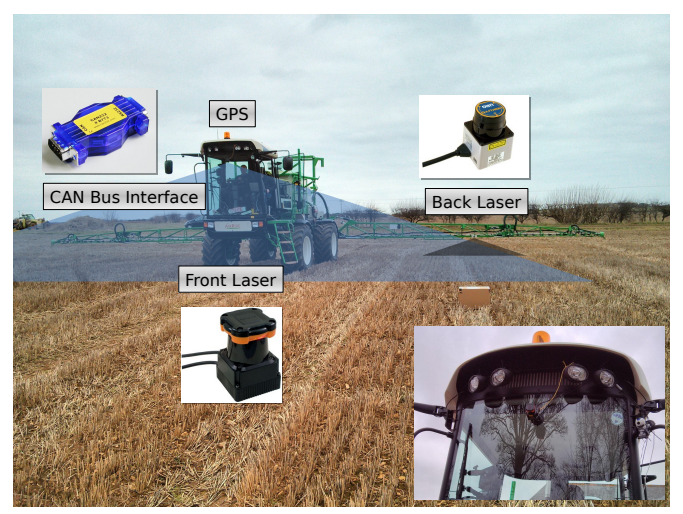

(a)

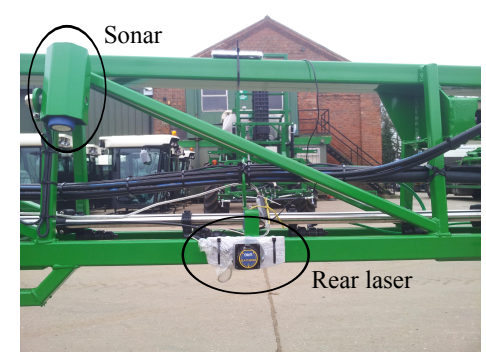

(b)

Fig. 2. a) Overview of the proposed system. b) Placement of the rear laser and sonar.

on each side. The current sensory system of the sprayer is composed of a set of ultrasonic sensors regularly distributed along the booms (every 5-6 meters) and pointing downwards so that they can measure the distance between the ground (or the crops) and the booms. The sonar data is provided through the internal CAN bus which also provides the information about the current configuration of the boom levelling platform including its height and incline angles.

In addition, the vehicle is equipped with a Trimble GPS receiver used for vehicle auto steering and variable-rate spraying application, providing global position measurements at a regular rate of $5 \mathrm{~Hz}$. The GPS receiver operates in a differential mode, receiving positioning corrections from a nearby base station thus achieving a theoretical accuracy of a few centimetres.

\subsection{Experimental Set-up}

To address the limitations of the current ultrasonic sensing system, we propose to use a laser scanner, attached to the front part of the sprayer's cabin to scan the ground surface in front of the vehicle (see Fig. 2(a)) and build a scrolling 3d map of the terrain as it approaches the sprayer. We used a Hokuyo UTM30LX outdoor laser scanner, which combines an affordable price and small size with good performance and relatively long range $(\sim 30 \mathrm{~m})$. We also employed an additional set of sensors for inspection, development and evaluation of the system. Thus, we attached a short-range laser scanner (Hokuyo URG-04LX) to the left spraying boom, pointing downwards and close to one of the ultrasonic sensors, so that the laser can scan the ground just below the boom (see Fig. 2(b)). A backup consumer-grade GPS was placed on the roof of the spraying vehicle in order to provide auxiliary positioning measurements used in initial stages of the system development.

In order to get the information from the sprayer's telemetrics, a CAN bus interface dongle has been connected to the vehicle's panel. This connection pro- 
vides access to the sonar readings and boom configuration parameters. All these components were connected to a main PC, a Dell XPSL502X laptop with an Intel CORE i7 processor running Ubuntu.

The required software was developed using the freely available Mobile Robotics Programming Toolkit [1]. The toolkit contains a set of libraries and applications covering the most common algorithms for mobile robot navigation, mapping and motion planning. It also provides a complete list of hardware and robotic sensors drivers including laser scanners, cameras and GPS. The toolkit has been used to collect data from the different sensors on the sprayer and to develop a stand alone application for off-line processing of the collected data, building the point maps from the laser scanner readings and visualisation, as well as measuring the errors between the data provided by the different sensors.

\subsection{Map Building}

The reconstruction of the terrain's surface was performed through the transformation of all the measurements captured with the laser scanner into one local reference frame $\mathbf{O}_{E}$ (see Figure 3), which is the main reference system of the working space and whose centre is placed at the starting position of the sprayer (i.e. the first position collected by the GPS). The vehicle's reference system $\mathbf{O}_{G 1}$ has its origin at the position of the sprayer's built-in GPS, with the $X$-axis pointing in the direction of the movement and the $Z$-axis pointing upwards. The reference systems $\mathbf{O}_{L 1}$ and $\mathbf{O}_{L 2}$ correspond to the front and the rear laser scanners, respectively, and have their $X$-axis pointing downwards. The $\mathbf{O}_{G 2}$ reference system is placed at the position of the auxiliary GPS. Finally, $\boldsymbol{T}_{V}$ stands for the pose (i.e. position and orientation) of the vehicle's reference system within the local reference system $\mathbf{O}_{E}$, and $\boldsymbol{T}_{L 1}$ and $\boldsymbol{T}_{L 2}$ for the poses of the two laser scanners with respect to the vehicle's reference system, respectively. Note that the vehicle's pose changes at each time step as the sprayer moves, whilst the last two are fixed since they are rigidly attached to the sprayer.

The process of building the map of the terrain from the laser data is accomplished as follows. Without loss of generality, we use the readings from the front laser in this explanation. Formally, let $r_{i}$ and $\alpha_{i}$ be the range and angle values measured by the front laser sensor for a certain scanned point $p_{i}$ on the terrain's surface. The coordinates of that point with respect to the laser scanner's reference system $\mathbf{O}_{L 1}$ are computed as $\mathbf{P}_{L 1}=\left[r_{i} \cos \left(\alpha_{i}\right), r_{i} \sin \left(\alpha_{i}\right), 0\right]$. Such a point can be further transformed with respect to the vehicle's reference system $\mathbf{O}_{G 1}$ through $\mathbf{P}_{G 1}=\boldsymbol{T}_{L 1} \oplus \mathbf{P}_{L 1}$, where $\oplus$ is the point-pose composition operator.

Finally, the coordinates in the local map of the scanned point $\mathbf{P}_{E}$ are computed in a similar way by applying again the composition operator with the pose of the vehicle within the local reference system $\boldsymbol{T}_{V}: \mathbf{P}_{E}=\boldsymbol{T}_{V} \oplus \mathbf{P}_{G 1}$. The set of points $\left\{\mathbf{P}_{E}^{i}\right\}$ referenced to the local system $\mathbf{O}_{E}$ computed this way constitutes the reconstruction of the the terrain's surface.

Note, that in order to build the map, it is necessary to estimate the vehicle's pose $\boldsymbol{T}_{V}$ at each time step, which can be achieved from the GPS readings, as well as to accurately measure $\boldsymbol{T}_{L 1}$ and $\boldsymbol{T}_{L 2}$, which is addressed next. 


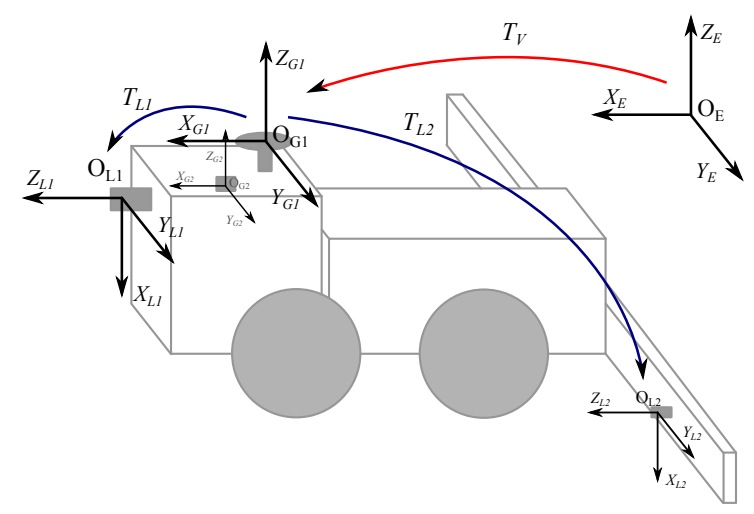

Fig. 3. All reference frames in the proposed system.

\subsection{Sensor Calibration}

Since the proposed system only relies on position data provided by GPS, the sensor calibration is a critical process to assure an accurate representation of the scanned surface. The estimation of the laser scanners' poses $\left(\boldsymbol{T}_{L 1}\right.$ and $\left.\boldsymbol{T}_{L 2}\right)$ with respect to the vehicle's reference system $\mathbf{O}_{G 1}$ has been performed as follows. First, an initial estimation of their positions was obtained by means of a standard measuring tape. Then the vehicle was driven through a field where three boxes of known size were placed on the soil (see Figure 4). The sprayer vehicle was driven so that the three boxes were scanned twice (one in each direction) with both lasers, and one of the boxes was scanned one more time in a perpendicular direction. These data have been used afterwards to refine the initial rough estimates by aligning the range scans corresponding to the boxes appearing in the reconstructed map. Figure 4(b) shows the effects of the initial calibration errors on the quality of the map reconstruction, while Figure 4(c) shows the final map after full calibration.

\section{Experiments}

\subsection{Datasets}

To evaluate the proposed system, we collected two data sets from different locations with different vehicle types and weather conditions (see Table 1 ). The estimated pose of the sensors for each data set is shown as a vector $[x, y, z, y a w$, pitch, roll $]$ where the translational part is expressed in meters and the rotational part in degrees. Data set \#1 was recorded while the vehicle was driven through a straight, hardened driveway while the spraying booms were suspended on an uncultivated field. Figure 5(a) shows the trajectory followed by the vehicle. This data set was used for finding the most convenient location of the sensors on the sprayer, verification of the initial set-up and assessing the laser's performance in 


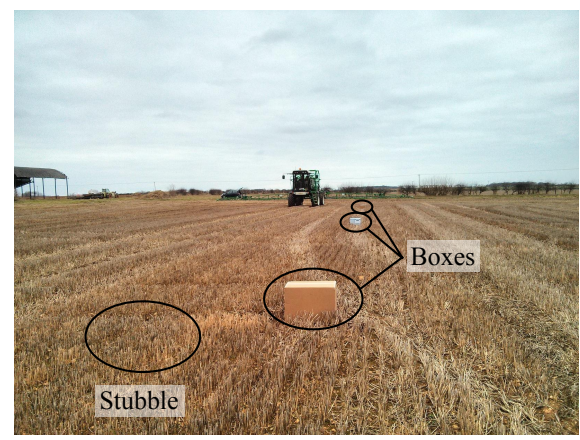

(a)

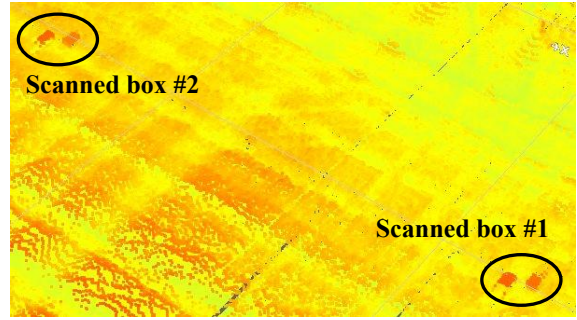

(b)

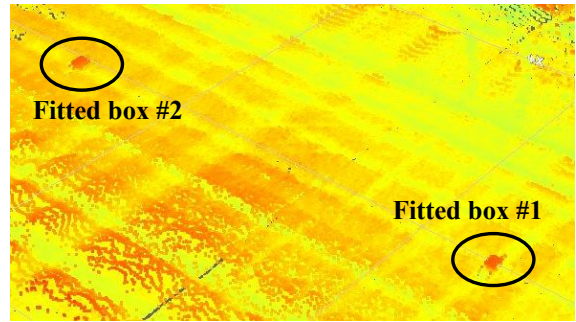

(c)

Fig. 4. The boxes used to perform sensor calibration (a). Detail of the map at the boxes (b) before and (c) after refining the calibration.

outdoor conditions and therefore the vehicle's telemetry data was not recorded. Data set \#2 was collected while the vehicle was driven on a field with short stubble left from a previous crop after harvesting. The main purpose of this data set was to assess the quality of the reconstructed map, but also for sensor calibration. Therefore three cardboard boxes were placed in known locations as depicted on Figure 4. This time the sprayer was driven following the path presented in Figure 5(b) while simultaneously recording data from the GPS receivers, the laser scanners and the sonar readings.

\subsection{Results}

Data set \#1 was collected mainly for development and testing of the system and therefore we only present qualitative results in the form of a reconstructed 3d map from the front laser readings (see Figure 6). One visible aspect in this map is a changing height pattern on both sides of the road corresponding to the rolling movement of the vehicle on uneven surface.

Data set \#2 was used to reconstruct and assess the quality of the $3 \mathrm{~d}$ map with all the sensors in operation. Figure 7 (a) shows the distribution of readings provided by the sonar sensors. Each green dot in the figure represents a location of a single sonar reading projected within the local reference system through the 
Table 1. Summary of the data sets.

\begin{tabular}{|r|l|l|}
\hline Parameter & Data set \#1 & Data set \#2 \\
\hline Date & $29^{\text {th }}$ of January, 2013 & $12^{\text {th }}$ of February, 2013 \\
\hline Weather & Cold, wet and cloudy & Cold, dry and cloudy \\
\hline Vehicle & Merlin & Air Ride \\
\hline Estimated pose: & & \\
Front laser & {$[0.99,-5.80,-3.20,0,90,0]$} & {$[1.75,0,-0.51,0,90,0]$} \\
Rear laser & {$[-5.59,8.90,-2.19,-90,84,-90]$} & {$[-5.03,5.89,-1.93,0,90,0]$} \\
\hline Total distance & $370.7 \mathrm{~m}$ & $290.4 \mathrm{~m}$ \\
\hline Total points & $5.7 \mathrm{M}$ & $4.5 \mathrm{M}$ \\
\hline
\end{tabular}

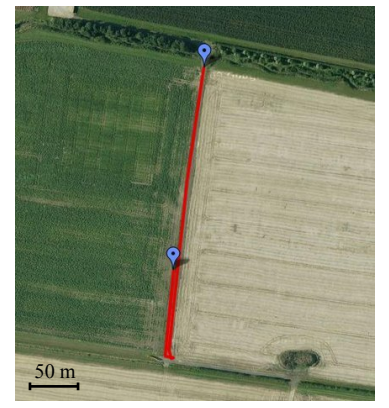

(a)

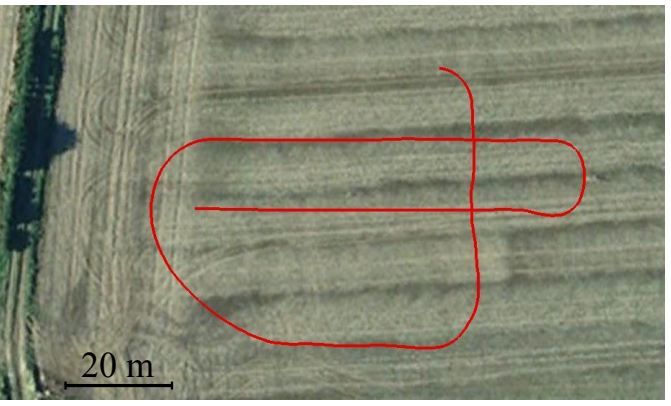

(b)

Fig. 5. A satellite view of the traversed path for a) Data set \#1 b) Data set \#2.

process described in Section 3.3. The points measured from the same sonar have been joined by a distinctively coloured line. As can be seen, the number of sonar measurements is scarce since the readings are only updated upon their change, which demonstrates the relatively low resolution of the sonar measurements. Figure 7(b) presents the map built with the rear laser, which was placed close to the left-centre sonar so that there exists an overlapping zone between the both sensors. It can be seen that a relatively narrow section of the terrain is reconstructed due to the low positioning and short range of the rear laser. In contrast, the map reconstructed using the front laser readings (see Figure $7(\mathrm{c})$ ) results in a dense cloud of points covering the terrain which forms an accurate model of the terrain. One visible artefact in the final $3 \mathrm{~d}$ map is a set of diagonal ridges caused by inaccuracies in pose estimation (mainly the pitch angle) due to the vehicle driving on uneven terrain.

A highlighted zone of the final map is presented in Figure 8(a) where the difference between the tracks of the vehicle and the areas with stubble can be noticed. The same area, represented as a $2 \mathrm{D}$ profile, is shown in Figure $8(\mathrm{~b})$ along with the median value in height, clearly showing the difference in profile between the track and the stubble (approx. $10 \mathrm{~cm}$ ).

In order to quantitatively assess the quality of the reconstructed surface, we have compared the scanned representation of one of the calibration boxes 


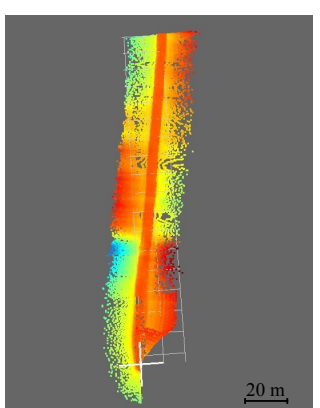

(a)

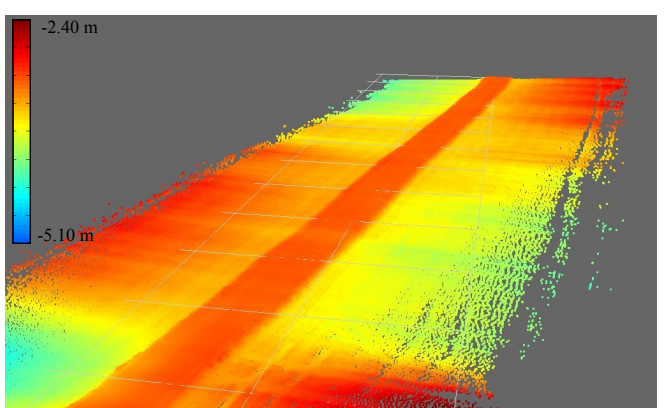

(b)

Fig. 6. The reconstructed map from Data set \#1 a) a top view, b) map detail.

with its real, physical size. This can be seen in Figure 9(a), where a wireframe model of the real box has been superimposed onto the point cloud. We then have measured the distance from three of the sides of the scanned box to the real sides and plotted these errors as histograms (see Fig. 9(b)). The average errors between the scanned model and the real box are $24 \mathrm{~mm}$ in height, and 43 and $55 \mathrm{~mm}$ in the lateral directions.

Finally, for comparison of different sensor types, we have computed the distance differences between the points measured by the sonar sensors and the maps reconstructed by each of the laser scanners. For that purpose, we have calculated the distance between the closest pair of readings from each sensor. Figures 10(a) and 10(b) show the histograms of distances between the readings from the leftcentre sonar only and the maps built from the front and the rear laser scanner, presenting mean distances of $44 \mathrm{~mm}$ and $56 \mathrm{~mm}$, respectively. The histograms of errors between the readings from all the sonars and the maps are presented in Figures 10(c) and 10(d), showing a mean distance of $56 \mathrm{~mm}$ for the front laser's map and $81 \mathrm{~mm}$ for the map built from the rear laser readings. Note that the results corresponding to the rear laser present higher mean distances because of the reduced data rate the sensor provides.

\section{Conclusions and Future Work}

In this paper, we presented a new application of laser range-finder mapping for use in agricultural spraying vehicles. The proposed approach results in dense $3 \mathrm{~d}$ maps of the terrain, providing a more detailed and accurate representation than the current sonar-based solution and a predictive capability for automatic boom control. Based on an assumed maximum vehicle speed of $15 \mathrm{~km} / \mathrm{h}$ and a distance between the front laser and the booms of 5 meters, we would therefore estimate that the proposed solution can provide an additional 1.2 seconds (minimum) of look-ahead time for the boom controller. In addition, only one sensor is needed to cover the full width of the sprayer with the booms unfolded in comparison with the current ultrasonic set-up consisting of five sensors. The results show 


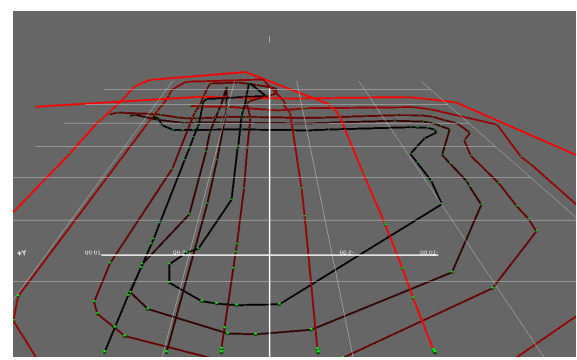

(a)

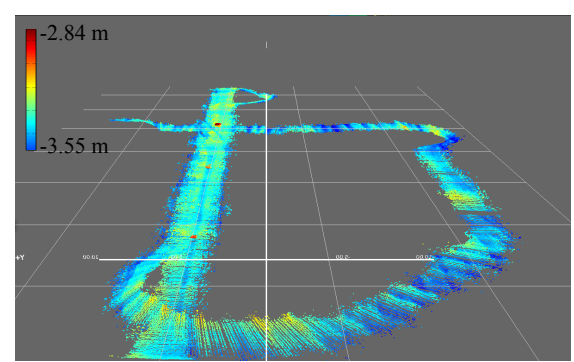

(b)

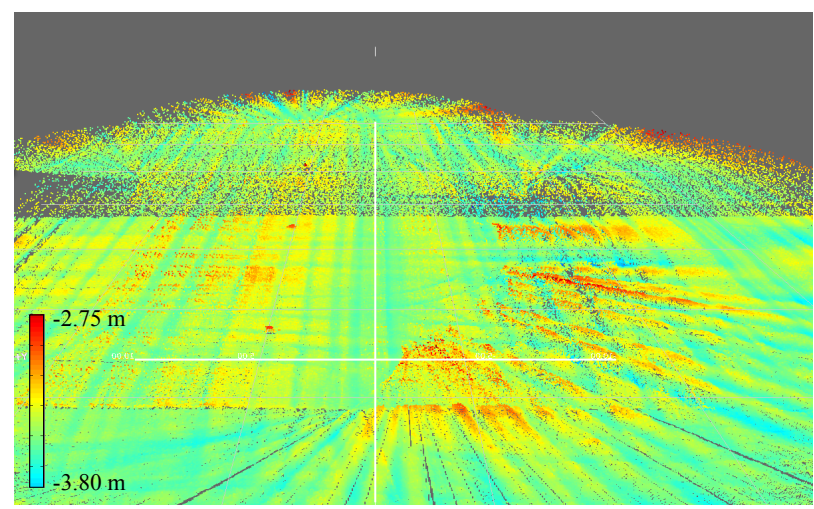

(c)

Fig. 7. The reconstructed map from Data set \#2: a) distribution of sonar readings, b) rear laser, c) front laser.

a mean distance between the estimated position of the ground computed from sonar and laser data of approximately $5 \mathrm{~cm}$.

The major problem affecting the accuracy of the reconstructed map is the estimation of the vehicle's pose in uneven terrain. The vehicle's turning angle can only be estimated from the GPS measurements at the moment, which leads to unreliable results on curves. Moreover, when the terrain presents potholes and irregularities under the sprayer's wheels, the shaking and vibrating movements introduce significant inaccuracies in the reconstructed maps. The use of an inertial measurement unit should address this issue as the full pose of the sprayer could then be estimated more accurately. To fully assess the characteristics of the proposed system, evaluation under different types of fields, crops and weather conditions (including strong sunlight) also has to be addressed in future.

This work represents a first step toward more autonomous solutions for agricultural spraying vehicles. Future work will consider a full closed-loop boom controller based on the proposed sensing principle. In addition, the proposed mapping system can lead to other applications including automatic detection of 


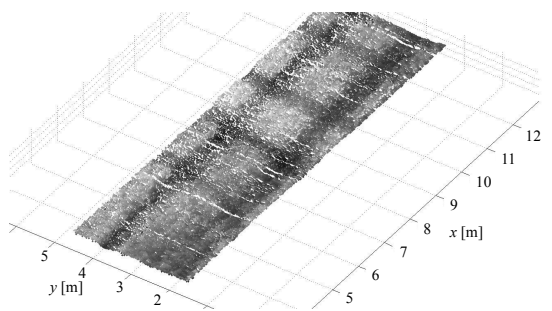

(a)

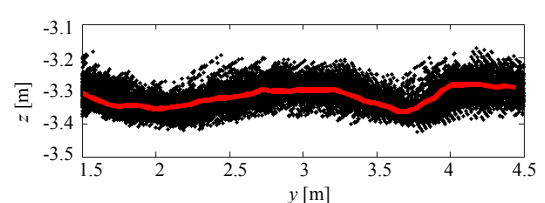

(b)

Fig. 8. (a) Detail of the map where the vehicle's tracks can be seen. (b) A 2D profile of the same area.

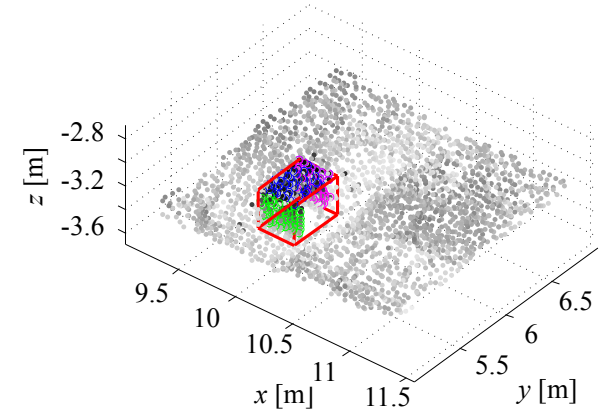

(a)

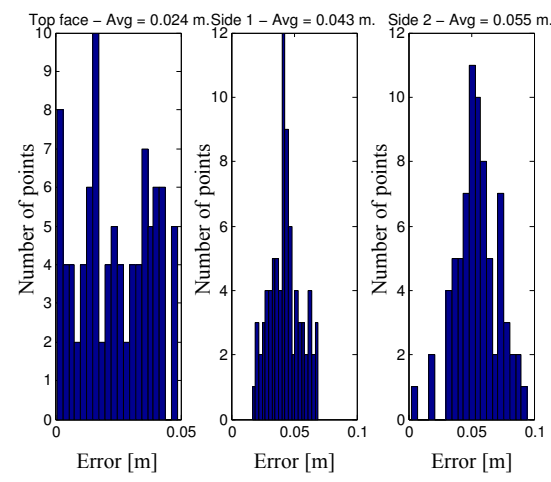

(b)

Fig. 9. The wireframe model of the test box superimposed onto the reconstructed point cloud (a). Histogram of errors between the readings and the actual box size in (b) the top face and $(\mathrm{c}, \mathrm{d})$ the lateral faces.

irregularities of the terrain, detection of tracks caused by the vehicle, or estimation of crop yields over time.

\section{Acknowledgment}

This project was supported by a BBSRC SPARK Award, in collaboration with Househam Sprayers Ltd.

\section{References}

1. MRPT framework. Website (2013), http://www.mrpt.org

2. Anthonis, J., Audenaert, J., Ramon, H.: Design optimisation for the vertical suspension of a crop sprayer boom. Biosystems Engineering 90(2), 153 - 160 (2005)

3. Biber, P., Weiss, U., Dorna, M., Albert, A.: Navigation system of the autonomous agricultural robot "BoniRob". In: Proc. IROS Workshop on Agricultural Robotics (2012) 


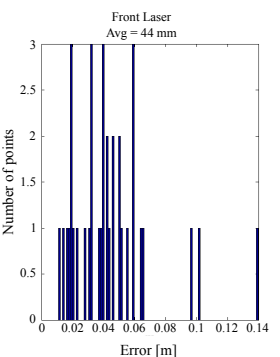

(a)

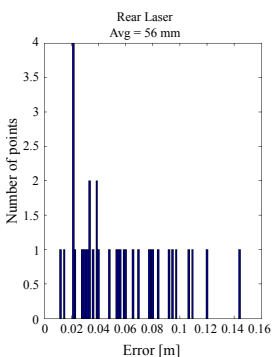

(b)

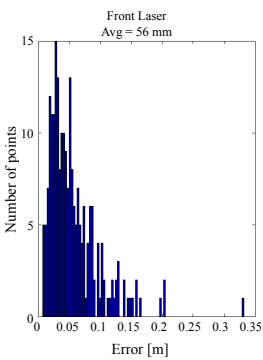

(c)

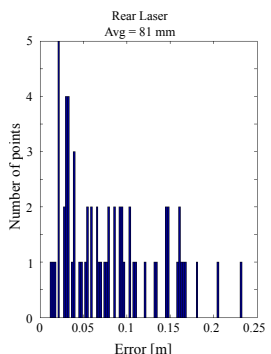

(d)

Fig. 10. Histograms of errors between a) left-centre sonar and front laser, b) left-centre sonar and rear laser, c) all sonars and front laser, d) all sonars and rear laser.

4. Jeon, H., Womac, A., Wilkerson, J., Hart, W.: Sprayer boom instrumentation for field use. Transactions of the ASAE 47(3), 659-666 (2004)

5. Magnusson, M., Lilienthal, A., Duckett, T.: Scan registration for autonomous mining vehicles using 3D-NDT. Journal of Field Robotics 24(10), 803-827 (2007)

6. Melese Endalew, A., Debaer, C., Rutten, N., Vercammen, J., Delele, M.A., Ramon, H., Nicolaï, B.M., Verboven, P.: Modelling the effect of tree foliage on sprayer airflow in orchards. Boundary-Layer Meteorology 138, 139-162 (Jan 2011)

7. Moorehead, S.J., Wellington, C.K., Gilmore, B.J., Vallespi, C.: Automating orchards: A system of autonomous tractors for orchard maintenance. In: Proc. IROS Workshop on Agricultural Robotics (2012)

8. Moreno, F.A., Gonzalez-Jimenez, J., Blanco, J.L., Esteban, A.: An instrumented vehicle for efficient and accurate $3 \mathrm{~d}$ mapping of roads. Computer-Aided Civil and Infrastructure Engineering (2013)

9. Nüchter, A., Lingemann, K., Hertzberg, J., Surmann, H.: 6d SLAM - 3d mapping outdoor environments. J. Field Robot. 24(8-9), 699-722 (Aug 2007)

10. Pedersen, S., Fountas, S., Have, H., Blackmore, B.: Agricultural robots - system analysis and economic feasibility. Precision Agriculture 7(4), 295-308 (2006)

11. Rovira-Más, F., Zhang, Q., Reid, J.F.: Stereo vision three-dimensional terrain maps for precision agriculture. Computers and Electronics in Agriculture 60(2), 133 $143(2008)$

12. Sun, J., Miao, Y.: Simulation and controller design for an agricultural sprayer boom leveling system. In: Measuring Technology and Mechatronics Automation (ICMTMA), 2011 Third International Conference on. vol. 3, pp. 245-248 (2011)

13. Thrun, S., et al.: Stanley: The robot that won the DARPA grand challenge. In: Buehler, M., Iagnemma, K., Singh, S. (eds.) The 2005 DARPA Grand Challenge, Springer Tracts in Advanced Robotics, vol. 36, pp. 1-43 (2007)

14. Walklate, P., Cross, J., Richards, G., Murray, R., Baker, D.: Comparison of different spray volume deposition models using lidar measurements of apple orchards. Biosystems Engineering pp. 253-267 (2002)

15. Wei, J., Salyani, M.: Development of a laser scanner for measuring tree canopy characteristics, phase 2: Foliage density measurement. Transactions of the ASABE 48(4), 1595-1601 (2005)

16. Wellington, C., Campoy, J. abd Khot, L., Ehsani, R.: Orchard tree modeling for advanced sprayer control and automatic tree inventory. In: Proc. IROS Workshop on Agricultural Robotics (2012) 